\title{
Medio ambiente, sustentabilidad y participación ciudadana: una mirada desde la sociología contemporánea
}

\author{
Salvador Orlando Alfaro \\ Universidad de Regina (Canadá)
}

RESUMEN: El desarrollo sustentable en su versión crítica incorpora una nueva relación entre sociedad y naturaleza; es decir, considera los problemas ambientales dentro de un sistema mayor que abarca las interacciones entre el ambiente natural, el ambiente construido y el ambiente social, donde el ser humano es indisociable de su entomo.

ABSTRACT: On its critical version, sustainable development is a concept that includes a new relation between nature and society. This concept considers that environmental problems belong to a larger system that includes the interactions between natural environment, constructed environment and social environment, in which the buman being can not be dissociated from bis environment.

\section{Introducción}

Considerando la relevancia que ha ido adquiriendo la cuestión medioambiental en el mundo, sus particulares características en América Latina y especialmente en nuestro país, es que resulta imperativo reflexionar sobre una problemática multicausal, contingente, socialmente 
emergente y compleja en su solución, producto, precisammente de la multiplicidad de factores que la han generado. De lo anterior resulta obvio que este escenario de complejidad y crisis social, que el sociólogo alemán Ulrich Beck denomina "la sociedad del riesgo", requiere de nuevos esfuerzos e interpreataciones desde la ciencia social.

En tal sentido, y tomando como punto de partida la contribución analítica de Beck, cuando hablamos de riesgos, discutimos de algo que no está a la vista, pero que puede hacer su aparición si no se toman ahora mismo cartas en el asunto. Y cuanto más amenazadoras son las sombras que se ciernen sobre el presente $-\mathrm{o}$ el anuncio de un futuro terribletanto más persistentes son las sacudidas que se pueden producir actualmente mediante la dramaturgia del riesgo. Una sociedad que se ve a sí misma como sociedad del riesgo, está en una situación de culposidad (para Beck en una situación pedadora) donde al menos se cuestiona o reflexiona sobre la posibilidad y deseabilidad de una vida mejor y de riesgos futuros que hay que evitar. En este autocuestionamiento se pone en movimiento, mediante los riesgos percibidos, la capacidad de acción de la sociedad, por cierto de la sociedad civil.

Indagando sobre las posibles salidas a la actual crisis socio-ambiental, nos encontramos con el paradigma de desarrollo sustentable, el cual incorpora una nueva relación entre ser humano, sociedad y naturaleza, comprendiendo que la naturaleza es la que sustenta y provee la base de recursos para la supervivencia de la humanidad, por consiguiente, todo deterioro ambiental disminuye la calidad de vida (Leff et al. 2000).

El desarrollo sustentable en su versión crítica incorpora una nueva relación entre sociedad y naturaleza; es decir, considera los problemas ambientales no como hechos aislados sino que los inscribe en un sistema mayor que abarca múltiples y complejas interacciones entre el ambiente natural, el ambiente construido y el ambiente social, donde el ser humano es indisociable de su entorno, el que comparte con otros seres vivos (ONU 1992). La naturaleza es la que sustenta y provee la base de los recursos para el desarrollo de la humanidad. Visto de otro modo, todo deterioro ambiental disminuye la calidad de vida. Por ello, se considera indispensable la participación ciudadana para poder avanzar hacia la sustentabilidad, ya que sin un actor consciente de los problemas que lo afectan, de sus potencialidades de cambio y de estrategias articuladoras para enfrentar estos desafios, la sustentabilidad queda supeditada a un nivel discursivo.

\section{4}

Realidad 106, 2005 
El desarrollo sustentable se propone como modelo de desarrollo que permitiría a las sociedades actuales y futuras mantener y/o elevar la calidad de vida, además de conservar y restaurar los recursos naturales desarrollando crecientes niveles de conciencia y sensibilidad y responsabilidad social. Lograrlo requiere la conjunción y participación de todos los sectores de la sociedad comprometidos a redefinir y construir una democracia con sustentabilidad.

De este modo aparece la participación ciudadana como factor clave para avanzar en la solución de los problemas ambientales, ya que su ejercicio contínuo permite incorporar en la toma de decisiones las opiniones de la comunidad, de manera que ésta pueda contribuir a la prevención y resolución de los conflictos ambientales. En este sentido, la conciencia ciudadana ambiental, y en general, el fortalecimiento de los valores como derechos humanos, la conservación ambiental y el derecho a participar en las decisiones que nos afectan directamente, son elementos fundamentales de una parcicipación comprometida con el medio ambiente.

En este artículo se abordan algunos de los más importantes elementos analíticos que actualmente son sujetos de discusión en torno a la problemática de la sustentabilidad y de la partipación ciudadana, relevando la acción que existe entre estos dos marcos conceptuales. Se argumenta teóricamente la necesaria concurrencia de ambos factores para poder enfrentar los problemas ambientales que en la sociedad del riesgo nos están alertando sobre la necesidad de buscar nuevos enfoques y miradas comprometidas con la búsqueda de soluciones a estos complejos problemas que estamos generando. Y tal como lo señala Beck, los problemas del medio ambiente no pueden ser enfrentados apelando solamente al cambio tecnológico y estudios científicos, sino que requiere de la generación de acuerdos y discusiones y de la participación de voces ciudadanas de alerta sobre el peligro (Beck 1992).

\section{La sustentabilidad como un nuevo paradigma de desarrollo}

No se puede desconocer el hecho que los actuales patrones de consumo sobre el medio ambiente y la base de los recursos lleva a la mayoría de los países de la periferia de la economía-mundo capitalista a mantener sus exportaciones (o su articulación dependiente a la economía globalizada) a expensas de sus recursos naturales y de la calidad ambiental con los correspondientes efectos sobre los ecosistemas y sobre las condiciones de la salud humana. Toda la naturaleza, toda la biósfera está al servicio de 
los aparatos de producción creados por las lógicas económicas pari satisfacer las condiciones de reproduccón cada vez más apremiantes articuladas al mismo tiempo, por relaciones sociales que corresponden : diferentes etapas de la acumulación de capital. Esa evolución cre: contradicciones y limitaciones que no son percibidas por la mayoría de li población (Schnaiberg y Gould, 1994).

De este cuestionamiento a la modernidad capitalista entendida coms progreso, se asume que no es posible desvincular los problemas del medic ambiente de los tradicionales problemáticas vinculadas al desarrollo, puestc que los primeros son la simple expresión de las insuficiencias del desarrollo Esta crisis se refiere al agotamiento de un estilo de desarrollo "ecológicament" depredador, socialmente perverso y políticament injusto" (Guimaraes 2000).

Por lo tanto, lo que se requiere es potenciar un nuevo paradigma di desarrollo, que coloque al ser humano en el centro del proceso di desarrollo, que considere el crecimiento económico como un procesc ecológicamente limitado, como uno de los medios para alcanzar mayore: niveles de bienestar humano y no como un fin en sí mismo; que protej: las oportunidades de vida de las generaciones actuales y futuras; y qui por lo tanto, respete la integridad de los ecosistemas sistemas naturale: que permiten la existencia del planeta.

De este modo, postular al desarrollo sustentable como paradigm: alternativo de desarrollo, implica un cambio en el propio modelo di civilización hoy dominante, parricularmentc en lo que se refiere al patrór de articulación ser humano-sociedad-naturaleza.

Este nuevo estilo de desarrollo deber ser ambientalmente sustentable er el acceso y uso de los recursos naturales, en la preservación de li biodiversidad, en la implementación de tecnologías limpias en los proceso: industriales y en un replanteamiento de los patrones de consumc occidentales. Socialmente sustentable, en la reducción de la pobreza, de lá desigualdades sociales y que promueva la justicia y la equidad social Culturalmente sustentable, en la preservación de la diversidad en el sentidc más amplio; es decir, en la preservación de valores, prácticas y símbolos di identidad de las culturas que coexisten en el presente periodo histónico; ) políticamente sustentable al empujar y radicalizar los procesos de reform: democrática y garantizar el acceso y la participación en la toma de decisiones

De esta forma, el desarrollo se plantea como un "paradigma d desarrollo" y no de crecimiento, porque establece un límite ecológicc 
temporal al proceso de crecimiento económico (en contraposición a la concepción dominante y comúnmente aceptada de que no es posible acceder al desarrollo sustentable sin crecimiento en la economía). El paradigma de la sustentabilidad parte de la base que el crecimiento, tal como lo hemos estado viviendo, constituye un componente intrínseco de la "insustentabilidad" del modelo actual. Por otro lado, es necesario poner de relieve que para que exista el desarrollo se requiere, más que una simple acumulación de bienes y servicios, cambios cualitativos en la calidad de vida de las personas, aspectos que, más que las dimensiones mercantiles, incluyen dimensiones sociales, culturales, estéticas y de satisfacción de las necesidades materiales y espirituales.

Pero no basta con que el desarrollo promueva cambios cualitativos en el bienestar humano y garantice la integridad ecosistémica del planeta para que sea sustentable. Es necesario señalar que especialmente en los países latinoamericanos, con graves problemas de pobreza, desigualdad y exclusion, los fundamentos sociales de la sustentabilidad suponen el postular como criterios básicos de la política pública los de la justicia distributiva, para el caso de los bienes económicos, y los de la universalización de cobertura, para las políticas globales de educación, salud, vivienda y seguridad social. Lo mismo se aplica en la sustentabilidad social, donde los criterios de igualdad de género, por ejemplo, se deben reconocer como un valor en sí mismo, y por lo tanto, por encima de consideraciones económicas.

El desarrollo sustentable, supone también la preservación de la diversidad en su sentido más amplio; es decir, la preservación de valores, prácticas y símbolos de identidad que determinan la integración nacional a traves de los tiempos (Sustentabilidad Cultural).

Otro elemento que está a la base de la sustentabilidad, es el fundamento político, que se encuentra estrechamente vinculado al proceso de reforma democrática y de construcción de ciudadanía, y busca garantizar la incorporación plena de las personas al proceso de desarrollo. Ello implica tanto una democratización de la sociedad como del Estado, donde se requiere el fortalecimiento de las organizaciones sociales y comunitarias, la redistribución de los recursos y de la información hacia los sectores sociales más vulnerables. Se asume la necesidad de capacitar a la ciudadanía para la toma de decisiones, lo que implica el desarrollo de estrategias que permican a la ciudadanía contar con un diagnóstico de su realidad para diseñar estrategias —en forma participativa- que aborden los problemas 
que enfrentan. Es decir, iniciar procesos de concientización sobre los problemas de la sustentabilidad a nivel nacional y local, y la necesidad de enfrentarlos de manera conjunta y propositiva.

Lo que une y da sentido a esta comprensión específica de la sustentabilidad, tal como ya se ha señalado, es la necesidad de una nueva ética del desarrollo. Ello dice en relación con la justicia social, con la justicia productiva y justicia distributiva. La primera se dirige a garantizar la condiciones que permiten un proceso redistributivo de la riqueza, especialmente orientado hacia los grupos mas vulnerables, la posibilidad real de estos grupos de satisfacer sus necesidades básicas acorde a las condiciones históricas actuales, y la existencia de una percepción generalizada de justicia y de tratamiento acorde a su dignidad y con sus derechos como seres humanos. La ética en cuanto materialización a través de la justicia distributiva se orienta a garantizar que cada gente reciba los beneficios del desarrollo conforme a sus méritos, sus necesidades, y su contribución al mejoramiento del conglomerado social (Schabecoff 1996).

El desarrollo sustentable implica por tanto una definición ética de la ciudadanía. Esto es, una opción por la equidad social, por la sustentabilidad ambiental y por la reforma democrática, que fortalece el posicionamiento ciudadano por la lógica del bienestar de las mayorias como factor de sustentabilidad y gobernabilidad, y fundamenta una visión crítica de la lógica neoliberal dominante presente en las actuales opciones hegemónicas de desarrollo. Permite, además, evaluar propuestas y estrategias de desarrollo desde una perspectiva de nación. Esto puede generar desde los ciudadanos un condicionamiento del desarrollo a los objetivos de la sustentabilidad, aportando a la construcción de una visión y una cultura para avanzar hacia las sociedades sustentables.

Dotar la sustentabilidad al desarrollo, requiere el fortalecimiento y democratización del Estado, y no - como lo proclaman los portavoces del neoliberalismo dominante- su abandono y sustitución por el mercado. Las dificultades provocadas por situaciones extremas de desigualdad social y de degradacion ambiental no pueden ser definidas como problemas individuales, sino que constituyen desafios sociales y colectivos. No se trata simplemente de garantizar acceso, vía mercado, a la educación, a la vivienda, a la salud, o a un ambiente libre de contaminación, sino de recuperar prácticas colectivas (solidarias) de satisfacción de necesidades.

Por ello es fundamental la concurrencia de actores capaces de movilizar estas propuesta, que si no es abordada en forma mancomunada desde 
diversos ámbitos de acción, tanto a nivel internacional, nacional y local, no es posible pensar en un avance hacia un modelo distinto al actual. El desafio institucional planteado por la crisis de sustentabilidad del desarrollo de nuestras sociedades se resume, en la democratizacion social (incluyendo las urgentes reformas al modelo económico dominante) y en la democratización del Estado. El primer objetivo supone el fortalecimiento de las organizaciones sociales y comunitarias, la redistribución de los recursos y de la información hacia los sectores socialmente marginados, el incremento constante de la información hacia los sectores socialmente marginados, el incremento constante de la capacidad de análisis de sus organizaciones y la capacitación para la toma de decisiones. El segundo, se logra a través de la apertura del aparato estatal al control ciudadano, de la reactualización de las prácticas políticas y de las organizaciones, así como la función específica de los procesos electorales y de la incorporación del concepto de responsabilidad política en la actividad pública.

Para que una sociedad sea considerada democrática y sustentable, debe tener como meta alcanzar la justicia social, y ello es posible en la medidad en que haya un cambio a nivel institucional, que éstas hagan viable y el ejercicio real de esos ideales a través de procesos sociales concretos. Con esto nos estamos refiriendo en primer lugar, al ejercicio pleno de los derechos mas elementales de la ciudadanía, tales como el derecho a reunirse, a pertenecer a organizaciones sociales y políticas, así como el acceso a la toma de decisiones (Pineiro, 2003). Pero debe trascender la dimensión individual, y pasar a formas colectivas de participación y representación, lo que permite el surgimiento de una acción común y positiva que adquiera sentido de decision colectiva.

La práctica democrática supone también que los derechos ejercidos por la ciudadanía en forma colectiva puedan asegurar su intervención en la toma de decisiones sobre las políticas de desarrollo, a nivel nacional y local. Para ello es indispensable definir el tipo y grado de autonomía de las organizaciones sociales (ambientales, sindicales, locales, etc.) para intervenir en el proceso de toma de decisiones, especialmente en lo que respecta a la orientación de estrategias económicas y de su viabilidad con respecto a la sustentablidad ambiental.

\section{Ciudadanía y sustentabilidad}

Con lo anterior nos introducimos a una dimensión fundamental de la sustentabilidad democrática, cual es la participación ciudadana. La idea 
de la ciudadanía para la sustentabilidad se refiere a la capacidad política y social de un colectivo para habilitar la democracia participativa y sus instituciones, lo que implica: crear las instituciones ciudadanas; desarrollar opinión pública y actores sociales deliberantes; organizar grupos que ejerciten en control social de las políticas de las instituciones gubernamentales y organismos multilaterales; promover la interlocución proactiva entre las redes de información y producción de conocimientos de los ciudadanos organizados; generar esferas públicas de negociación de las agendas estatales; asegurar la construcción de políticas públicas que expresen las demandas y propuestas de la sociedad civil y de los gobiernos locales, a la vez que se institucionalizen mecanismos para la resolución de conflictos; crear los espacios políticos que permitan el ejercicio y la consecución de la reforma social entre otros.

Bajo el prisma de la ciudadanía para la sustentabilidad, se plantea la necesidad de redefinir lo público como un ámbito en el cual se procesan nuevos contratos para sustentar la acción del Estado de manera legítima e innovadora, lo que implica ampliar y perfeccionar los mecanismos de participación ciudadana, establecer canales de expresión democrática directa y reconocer la facultad y los derechos que tienen las organizaciones ciudadanas para fiscalizar y pedir cuentas al al gobierno sobre el cumplimiento de sus compromisos y resultados (Shabecoff 1996: 43-47).

De este modo, la ciudadanía ambiental (ciudadanía por la sustentabilidad) se articula a partir de las siguientes dimensiones: En primer lugar, como atributo de una gobernabilidad ambiental que asume la construcción participativa de agendas de sustentabilidad, y el seguimiento y evaluación de las políticas públicas que afectan la sustentabilidad de las estrategias de desarrollo y no sólo de las políticas ambientales, y se materializa en las grandes y pequeñas decisiones políticas como última prueba sobre la efectividad del ejercicio de ciudadanía. $\mathrm{Y}$ en segundo lugar, se manifiesta como "asociativismo" ciudadano, organizado en diversas modalidades, que delibera en la esfera pública acerca de los problemas que los afectan (Neefjes 2000).

La ciudadanía por la sustentatblidad representa una expresión de una nueva ciudadanía, fuertemente arragaida en el ámbito de lo público, que ciertamente no es patrimonio exclusivo del Estado, ni se agota en la esfera gubernamental, debiendo ser entendido como aquello que es de todos, lo que nos pertenece como comunidad humana y de lo cual todos somos responsables. 
El desafío de la ciudadanía por la sustentabilidad exige avanzar hacia una nueva generación de políticas públicas, lo cual representa una tarea estratégica que puede y debe ser asumida por las diversas organizaciones de la sociedad civil comprometidas con la sustentabilidad.

Por último, cabe señalar, que el desarrollo sustentable como meta a alcanzar, como paradigma en construcción, es un proceso que requiere de la concurrencia de múltiples y diversos actores (del Estado y sociedad civil en todas sus expresiones y formas de organización), donde las prioridades de desarrollo y progreso social se definan en conjunto y donde los ciudadanos puedan incidir en la toma de decisiones, y para ello es fundamental la democratización del Estado, como de las formas y espacios de participación de los ciudadanos. En este sentido, el desarrollo sustentable provee un marco conceptual amplio que debe ser abordado con las políticas públicas (económicas, sociales, medioambientales y culturales) al interior de cada Estado-Nación.

Entonces, para poder avanzar hacia el desarrollo sustentable, es indispensable mirarlo como un proceso de cambio social, tecnológico y cultural, con metas en un corto, mediano y largo plazo y con un compromiso real de avanzar hacia la equidad social y la superación de la pobreza estructural; avanzar hacia la preservación y uso sostenible de los recursos naturales y la preservación y uso sustentable de los recursos naturales y la preservación de la biodiversidad; avanzar en la preservación de la diversidad cultural (preservación de valores, prácticas y símbolos de identidad de las diversas culturas que coexisten en las sociedades); y avanzar en la "radicalización" de la democracia con el objetivo de garantizar el acceso y la participación de los todos los sectores componentes del Estado-Nación en la toma de decisiones.

4. Participación ciudadana: Una estrategia para avanzar hacia la sustentabilidad

La problemática de la participación recorre todo el arco politico, y es reivindicada por corrientes tan diversas como el neoliberalismo, el neocontractualismo, la socialdemocracia, el marxismo en sus distintas interpretaciones, y en general en todas las formas de populismo. "Del mismo modo esta palabra se ha convertido en la referencia obligada de planes y programas de desarrollo, de manifiestos ideológicos y de campañas presidenciales" (Hopenyan 1989).

681 
Con lo anterior, to que se quiere enfatizar es que no existe una sola definición de participación. Esta adquiere variadas y múltiples dimensiones y configuraciones dependiendo del contexto en la cual se sitúe. Es así como existen diferentes definiciones de participación (Alfaro 2001).

Cabe destacar, primero, el discurso neoliberal representado por el propio Banco Mundial, para el cual la participación consiste en "movilizar el potencial de los pobres" para reducir su pobreza y vulnerabilidad. Esto implica reconocer y promover los activos que los pobres tienen en su hogar, en sus mujeres y en sus relaciones comunitarias. Esto presupone asumir que entre la dimensión económica y la social de la vida existe una relación metabólica inseparable (son como inhalación y exhalación en el proceso de respirar), de modo que el "poder de los pobres" podría acrecentarse potenciando la inhalación social para lograr éxitos en la exhalación económica. "El aumento de los outputs económicos a partir de los inputs sociales permitiría resolver gran parte del problema material de la pobreza y parte del problema democrático de la participación" (Harris 2002: 77). Visto así, el progreso efectivo se logra potenciado al pobre, al débil y al vulnerable para que se transformen en los productores de su propio bienestar y no en meros recipientes de la caridad o beneficiarios de la ayuda pública (World Bank 2002: 10-17).

Es evidente que el Banco Mundial restringe el concepto de participación a la movilización del activo social de los pobres (que no se podrían movilizar sin participación), pero a la vez reduce el concepto de probreza a sus contenidos dinámicos, sustrayendo del mismo sus contenidos pasivos (los factores estructurales que los generan). La exclusión de las dimensiones estructurales de la pobreza deja por tanto el concepto de participación sin ninguna connotación política.

La acción que propone el Banco Mundial no envuelve necesariamente ni completamente al Estado, dado que debe ser una acción privada, estrictamente técnica (no política) y limitada al tratamiento casuístico del problema. A este efecto, el Banco promovió la creación de una red mundial de Fondos Sociales -financiado en gran parte por la misma institución que administra la estrategia, diseña los programas concretos de acción y evalúa los resultados. El Banco promueve la reorganización del vínculo entre Estado y Sociedad desde la acción privada del mercado (las agencias ejecutoras compiten mercantilmente entre sî) lo que tiene un fuerte impacto en el caso de estos programas, al excluir de un modo directo la responsabilidad del Estado. 
En suma, para el Banco Mundial, la participación no es ni puede convertirse en práctica "política" (y menos en un poder político), sino permanecer todo el riempo como una práctica de "empresarialidad". Por tanto, se trata de una forma de incoporar los pobres al Mercado, no al Estado. (Harris 2002:81).

En este análisis, la participación se centra en el individuo, en tanto sujeto inalienable, que mediante su capacidad de gestionar proyectos "participarivos" puede acceder a fondos que le permitan "entre paréntesis" superar su condición de pobreza. Aquí de ninguna manera se observa un sentido potenciador de cambios vía la participación, más bien se tiende a mantener el status quo.

Otra visión de la participación es la que sostienen las agencias internacionales como los Programas de las Naciones Unidas para el Desarrollo (PNUD) y sobre el Medio Ambiente (PNUMA), donde el concepto aparece asociado al poder.

En una publicación auspiciada por el PNUD, James Midgley sostiene: "para que pueda haber una auténtica participación, es necesario que la gente local se libere de controles externos. Ello supone independencia tanto política como económica. Para tener poder se requiere autosuficiencia económica y también implica la creación de instituciones que permitan a la gente local la toma de decisiones" (1993: 210-211).

Mary Racelis por su parte, sostiene que "las estrategias de desarrollo social alternativo promueven el conferimiento de poder al pueblo en vez de perpetuar las relaciones generadoras de dependencia tan características de los enfoques de la cima a la base. Un programa que dé poder a la población, promoverá un proceso evolutivo nutrido por el acuerdo mutuo entre los agentes del desarrollo y la población. A través del conferimiento del poder, se da la tónica para un desarrollo (alternativo) genuinamente centrado en el pueblo (1993: 246-247).

Dotar el concepto de participación con la dimensión del poder equivale, definitivamente, a cambiar el énfasis desde el "pobre, el débil y el vulnerable" hacia el ciudadano y las comunidades locales, y desde la política transitoria referida a los bolsones residuales de pobreza hacia aquellas atingentes a los problemas de fondo que aquejan el modelo neoliberal.

Según Goulet (1989), la participación se construye en el acto mismo de conquistar, es un efecto ontogénico de la conquista de un derecho; de alí que cuando esa condición intenta donarse, se reemplaza la decisión y 
la iniciativa y, por lo tanto, los usuarios pueden llegar a incorporarse en los beneficios de las políticas, pero no constituye en sí participación real. En esta misma línea se podría caracterizar a la participación como sustantiva y real, aquella que se refiere al poder y a la toma de decisiones. $\mathrm{Y}$ a la diferencia de las anteriores, esta apunta al real sentido que debiera tener la participación; es decir, aquí la participación estaría asociada al control sobre el sentido de las decisiones y a la constitución de sujetos que se conforman en ese proceso de decidir y emprender acciones tendientes al mejoramiento de su calidad de vida.

En este enfoque de participación —que es distinta de la estrictamente liberal- se especifica así en el sentido de las decisiones y en las cuales participar, apunta a crear relaciones y estructuras más equitativas y humanas en los diversos niveles en que esas acciones tienen efecto (democratización), y construir sujetos. No opone el interés individual al interés colectivo y como lo demuestran múltiples testimonios de sujetos populares, los individuos crecen y se realizan como personas cuando se incorporan libremente a un grupo de acción, donde en vista de una práctica transformadora, participa de una identidad colectiva (Rahman 1990). En última intancia la participación real apunta al crecimiento de las personas y no sólo al crecimiento de las cosas, es un valor de justicia y autodeterminación.

Un rasgo esencial de la participación real es que esta sea informada, lo cual implica accesso público y equitativo a los antecedentes necesarios para ejercer los derechos y deberes ciudadanos. Reconociendo que la información otorga poder, representa de este modo, un recurso fundamental para asegurar la participación ciudadana responsable, efectiva y oportuna a nivel nacional y local. La disponiblidad de información simple y oportuna al alcance de los ciudadanos y sus organizaciones, pasa a ser un requisito de una participación ciudadana efectiva y responsable cuando los espacios democráticos se hayan construidos. Sin embargo, en sociedades periféricas y altamente estratificadas, existen grandes asimetrías en el acceso y uso de la información por parte de los ciudadanos y sus organizaciones respecto a aquella que pueda manejar el Estado y los grupos de poder económico locales y transnacionales, lo cual plantea el desafío de crear los mecanismos institucionales para garantizar una gestión transparente de los fondos y programas, poniendo al alcance de los ciudadanos y la sociedad civil los antecedentes necesarios para que puedan ejercer una participación real y no solo funcional. 
De esta forma, la búsqueda de la sustentabilidad nos remite a una participación multidimensional de las personas y comunidades, expresadas en múltiples ciudadanías que es necesario construir y desplegar, en el marco del ejercicio de derechos y obligaciones para una vida más humana, especialmente para quienes enfrentan condiciones de pobreza y vulnerabilidad como consecuencia de procesos de desarrollo capitalistas excluyentes y autoritarios.

La presencia de los ciudadanos en los asuntos públicos es una condición necesaria para alcanzar la gobernabilidad democrática. A medida que el ejercicio del poder está más legitimado en la voluntad obtenida a través de un consenso social, mayores son las posibilidades de hacer visibles las aspiraciones de la sociedad civil. Puede decirse que la realización progresiva de los derechos humanos está en relación directamente proporcional al aumento de la calidad de la participación ciudadana. Sin embargo, prevalece en nuestras sociedades una marcada exclusión de la mayoría poblacional, especialmente de los grupos populares, respecto a la toma de decisiones sobre los asuntos públicos; de manera que el consenso se reduce a minorías no representativas de la pluralidad de intereses encarnados en la sociedad civil. Una de las razones primarias para tal marginamiento, se desprende de la ausencia de información, e interiorización de la ciudadanía, sobre los límites y los alcances del marco juríco democrático-liberal en cuanto al reconocimiento formal de los espacios de participación. Por lo tanto, la presencia de los ciudadanos en las decisiones que los afectan, tanto individual como colectivamente, precisa una adecuación funcional, en tanto facilite el acceso y la conformación no excluyente de centros de decisión a partir del nivel local, que en última instancia, también sean capaces de incidir en la formación de la voluntad sobre las decisiones que exceden el ámbito del Estado-Nación (Gill 1995).

Se puede deducir de lo anterior, que la participación es un proceso de generación de conciencia crítica y propositiva de los ciudadanos. En verdad, se trata de un factor clave en el proceso de empoderamiento de los grupos sociales. La participación ciudadana no es realmente efectiva mientras no vaya modificando y ampliando las relaciones de poder. EI fin de la participación ciudadana no es tanto ella en sí misma, por cuanto que, la finalidad que debería perseguir es la mejora sostenible de las condiciones de vida de los más amplios sectores de la sociedad.

Es importante, a estas alturas, enfatizar que la participación es un componente esencial de la reforma demcrática, puesto que la participación 
permite concretar el ejercicio de la ciudadanía en sus diferentes dimensiones: civil, política y social. Dentro de esta última dimensión deben incluirse, como una contribución a la radicalización democrática, el derecho al empleo, a la seguridad física, a los servicios básicos, a la protección del medio ambiente y del patrimonio histórico y cultural sobre el que se contruye la identidad.

La participación ciudadana es la clave para transformar el espacio de lo estatal en un espacio público y contribuir a crear condiciones para que efectivamente se pueda construir y consolidar la reforma democrática; la cual se constituye como la necesaria contraparte política de la reforma económica y social.

\section{Consideraciones finales}

Se puede percibir en el concepto crítico de desarrollo sustentable un cuestionamiento de fondo a la racionalidad de la civilización moderna, donde la racionalidad capitalista ha generado un creciente proceso de racionalización formal e instrumental, queha moldeado todos los ámbitos de la organización burocrática, los metodos científicos y los aparatos jurídicos e idelógicos del Estado. El desarrollo sustentable no sólo plantea la necesidad de introducir reformas sustanciales al Estado, de incorporar nuevas normativas al comportamiento económico, de legitimar los nuevos valores éticos y de producir nuevas tecnologías para controlar los efectos contaminantes y disolver las externalidades sociales y ecológicas generadas por los impactos ambientales. El desarrollo sustentable cuestiona los beneficios y las posibilidades de mantener una racionalidad social fundada en el cálculo económico de corto plazo y las ganancias, la formalización, control y uniformización de los comportamientos sociales y la eficiencia de sus medios tecnológicos, que han inducido un proceso de degradación socio-ambiental, socavando las bases-mismas del proceso económico y minando los principios de equidad social y dignidad humana (Kay 1996).

En un sentido propositivo, la sustentabilidad abre así nuevas perspectivas al desarrollo, descubriendo nuevos potenciales ecológicos, tecnológicos y sociales, y planteando la transformación de los sistemas de producción, de valores y de conocimiento de las formas de organización social, para construir una relación diferente entre el ser humano la sociedad y la naturaleza.

Finalmente, junto con la generación y actualización del conocimiento de las ciencias sociales sobre sustentabilidad y progreso social, es 
imprescindible integrar aportes y prácticas de actores sociales que estan desarrollando estrategias de desarrollo sustentable, tendientes a generar cambios positivos en su entorno, condiciones y calidad de vida. Ello, sin duda, plantea la necesidad de "volver al actor" (Long 2001) y desde ahí aportar una mirada a sus prácticas en el sentido de retroalimentarlas, reforzarlas y enriquecerlas, a la vez nutrimos con sus experiencias de la vida cotidiana, saberes, prácticas, metodologías y significaciones.

\section{REFERENCIAS}

Alfaro, S.O. (2001) "Desarrollo Participativo y la Apropiación de la Agencia: Una Exploración Sociológica", Realidad 84. 807-817.

Beck, U. (1992) Risk Society: Towards a New Modernity. Londres: Sage.

Gill, S. (1995) "The Global Panopticon: The Neoliberal State, Economic Life, and Democratic Surveillance", Altematives 20(1). 1-49.

Goulet, D. (1989) "Participation in Development: New Avenues", World Development 17 (2). 165-78.

Guimaraes, R. (2000) "Participación, democracia y desarrollo sustentable", Quiroga, R. (ed.) Participación y desarrollo sustentable. Serie Documentos. Santiago de Chile.

Harris, J. (2002) Depoliticizing Development: The World Bank and Social Capital. Londres: Anthem Press.

Hopenhaym, M. (1989) "La participación y sus motivos", Revista Acción Crítica 24 y 25, CELATS, Lima.

Kay, M. (1996) Environmentalism and Cultural Theory. Londres: Routledge.

Leff, E. (ed.) (2000) La Complejidad Ambiental. Mexico: Siglo XXI.

Long, N. (2001) Development Sociology: Actor Perspectives. londres: Routledge.

Midgley, J. (1993) "La política social, el Estado y la participación de la comunidad", Kiksberg, B. (comp.) La Pobreza: un problema impostergable. Mexico: CLAD. 210-211.

Neefjes, K. (2000) Environments and Liveliboods: Strategies for Sustainability. Londres: Oxfam Publishing. 
Organización de las Naciones Unidas [ONU] (1992) Informe sobre la Conferencia de las Naciones Unidas sobre Medio Ambiente $y$ Desarrollo. Programa 21. Rio de Janeiro, Brasil.

Piñeiro, D. (2003) "Sustainability and Democratization in Latin American Rural Societies, Sociologias 10. 26-34.

Racelis, M. (1993) "Movilizando a la población para el desarrollo social", Kiksberg, B. (comp.) La pobreza: un problema impostergable. Mexico: CLAD. 246-247.

Rahman, M, (1990) "People's Self-development", Community Development Joumal Vol. 24 (4). 307-14.

Shabecoff, P. (1996) A New Name for Peace: International Environmentalism, Sustainable Development and Democracy. Londres: University Press of New England.

Shnaiberg, A. y Gould, K, (1994) Environment and Society: The Enduring Conflict. Nueva York: St. Martin's.

World Bank (2002) Empowerment and Power Reduction. Washington DC: The World Bank. 